\title{
Comportamento reológico de méis de florada de silvestre
}

\author{
Alexandre J. de M. Queiroz ${ }^{1}$, Rossana M. F. de Figueirêdo', Claudécia L. da Silva² \& Mario Eduardo R.M.C. Mata ${ }^{1}$
}

\begin{abstract}
RESUMO
Estudou-se o comportamento reológico de méis de Apis mellifera produzidos no estado do Piauí, no semi-árido brasileiro, elaborados com florada predominante de silvestre (Serjania glabrata). As amostras foram coletadas ao longo do ano 2000, a partir de coletas realizadas por apicultores, associações e cooperativas de apicultura da região abrangida pelos municípios de Picos, Itainópolis, Vera Mendes e Isaías Coelho. Após as coletas as amostras passaram pelos processos de centrifugação, filtragem e decantação. As medidas reológicas foram feitas nas temperaturas de 20 a $40{ }^{\circ} \mathrm{C}$ utilizando-se um viscosímetro Brookfield, modelo RVT. As leituras de velocidade de rotação e torque foram transformadas em valores de taxa de deformação e tensão de cisalhamento. Os dados de taxa de deformação e tensão de cisalhamento foram ajustados pelas equações da Leida-Potência e de Herschel-Bulkley. As amostras apresentaram comportamento pseudoplástico. As equações se ajustaram adequadamente aos resultados experimentais de tensão de cisalhamento em função da taxa de deformação. O aumento de temperatura reduziu a viscosidade aparente em níveis próximos de $80 \%$. Os valores de viscosidade aparente foram bem ajustados por uma equação do tipo Arrhenius.
\end{abstract}

Palavras-chave: Apis mellifera L., Serjania glabrata, viscosidade

\section{Rheological behavior of honey from Serjania glabrata flowers}

\begin{abstract}
The rheological behavior of Apis mellifera honey produced in the State of Piauí, in the Brazilian semi-arid, obtained with predominance of Serjania glabrata flowers was studied. The samples were collected in the year 2000 by beekeepers and associations and cooperatives of beekeepers in the area covered by the municipal districts of Picos, Itainópolis, Vera Mendes and Isaías Coelho. The samples were centrifuged, filtered, decanted and the rheological measures at temperatures of 20 to 40 ${ }^{\circ} \mathrm{C}$ using a Brookfield Viscometer RVT model were made. The readings of rotation speed and torque were transformed in shear rate and shear stress values. The data of shear rate and shear stress were fitted by the potential Law and Herschel-Bulkley models. The samples presented pseudoplastic behavior. The potential and Herschel-Bulkley models fitted the experimental results appropriately. The increase in temperature reduced the viscosity by approximately $80 \%$. The apparent viscosity values fitted well with an Arrhenius type equation.
\end{abstract}

Key words: Apis mellifera, beekeeping, viscosity

1 UAEAg/ UFCG, Av. Aprígio Veloso 882, CP 10.017, CEP 58109-970, Campina Grande, PB. Fone: (83) 3310-1548. E-mail: alex@deag.ufcg.edu.br 2 R. São Francisco 307, CEP 646000-000, Picos, PI. Fone: (89) 422-1902. E-mail: claudecia@yahoo.com 


\section{INTRODUÇÃO}

A Região Nordeste ocupa o segundo lugar na produção nacional de mel, tendo atingido $7.967 .658 \mathrm{~kg}$ de mel em 2003, enquanto o Piauí se sobressai como maior produtor da região (IBGE, 2003). A cultura do cajueiro oferece suporte para a produção apícola piauiense, notadamente no segundo semestre do ano, proporcionando grandes colheitas de mel da flor do cajueiro e do pseudofruto (Alcoforado Filho, 1998); no entanto, os méis piauienses são elaborados de forma predominante a partir da flora nativa, em que as abelhas utilizam néctar de flores de espécies endêmicas, como marmeleiro (Croton sonderianus Müll. Arg.), jitirana (Hyptis suaveolens), camaratuba (Cratylia mollis Mart. ex Benth), angico de bezerro (Piptadenia moniliformis) e silvestre (Serjania glabrata) entre outras. A matéria-prima fornecida por essas espécies confere, ao mel produzido no local, características únicas, uma vez que, como afirma Crane (1983), o aroma, sabor e a cor do produto, variam em função da sua origem floral. Para fins de comercialização o mel pode ser classificado conforme sua origem botânica (Brasil, 2000). De acordo com Crane (1983) e Campos (1987) frutose e glicose representam aproximadamente $70 \%$ dos açúcares do mel mas o percentual de açúcares varia em função da matéria-prima utilizada para sua elaboração, que se pode constituir não apenas de néctar mas, também, de outras substâncias açucaradas, como suco de frutas em decomposição ou resultantes da transpiração das plantas (Marques \& Wiese, 1983). Balbach \& Boarim (1992) afirmam que qualquer mel depende do tempo, solo e outros fatores mas principalmente das fontes vegetais dos quais é derivado. A fonte utilizada para elaboração do mel responde ainda pelo teor de vitaminas, pigmentos, enzimas, minerais, ácidos orgânicos, aminoácidos, proteínas, ácidos graxos, hormônios de crescimento e de enraizamento de plantas (Hooper, 1981); além disso, a consistência e a textura são igualmente influenciadas pela matéria-prima, em alguns casos como conseqüência do teor e do tipo de proteína, que confere a alguns méis características gelatinosas ou, ainda, reduz a tensão superficial, tornando-o mais propenso a reter bolhas de ar e formar espuma (Crane, 1983).

A consistência do mel, característica conferida pela viscosidade tem, como em outros alimentos, importância industrial e comercial. Industrialmente, maiores viscosidades implicam em maiores custos na centrifugação, decantação e misturas, ocasionados por acréscimos nos tempos de operação e gastos energéticos. Segundo Ibarz et al. (1996) o comportamento reológico dos alimentos fluidos é usado como medida de qualidade e também na elaboração de projetos, avaliação e operação de equipamentos e processos. Do ponto de vista comercial, a qualidade do mel é associada, pelo consumidor, ao comportamento reológico, que identifica o mel puro como um produto de viscosidade elevada. Estudou-se, neste trabalho, o comportamento reológico de méis de Apis mellifera, produzidos no estado do Piauí, elaborados com florada predominante de silvestre (Serjania glabrata) nas temperaturas de $20,25,30,35$ e $40{ }^{\circ} \mathrm{C}$.

\section{MATERIAL E MÉTODOS}

Os méis utilizados nos ensaios provinham da microrregião piauiense, abrangida pelos municípios de Picos, Itainópolis, Vera Mendes e Isaías Coelho. As amostras foram obtidas a partir de coletas realizadas por apicultores, associações e cooperativas de apicultura no decorrer do ano 2000.

O material foi identificado, quanto à origem botânica, de acordo com as informações dos apicultores, recebendo rótulo com o nome da localidade onde foi coletado. As amostras foram centrifugadas, filtradas, decantadas e acondicionadas em recipientes apropriados e, assim embaladas, foram encaminhadas ao Laboratório de Armazenamento e Processamento de Produtos Agrícolas, na Universidade Federal de Campina Grande, PB, onde foram armazenadas para a utilização nos ensaios reológicos.

As medidas reológicas foram feitas com as amostras nas temperaturas de $20,25,30,35$ e $40{ }^{\circ} \mathrm{C}$ utilizando-se um viscosímetro Brookfield, modelo RVT, fabricado por Brookfield Engineering Laboratories, EUA. Utilizaram-se velocidades de rotação de 0,5, 1,0, 2,5, 5,0, 10, 20, 50 e $100 \mathrm{rpm}$. As medições eram realizadas em volumes de aproximadamente $500 \mathrm{~mL}$ de mel, cujas temperaturas, acima citadas, eram controladas em banho termostático. Quando a amostra atingia a temperatura desejada realizavam-se as leituras de torque, que eram efetuadas após um tempo de $30 \mathrm{~s}$ de deformação, para evitar erros induzidos por possíveis efeitos de tempo. Os experimentos foram feitos em sextuplicata, efetuando-se este procedimento em todas as amostras.

As leituras de velocidade de rotação e torque foram transformadas em valores de taxa de deformação e tensão de cisalhamento, mediante o uso da metodologia proposta por Mitschka (1982). Os dados de taxa de deformação e tensão de cisalhamento foram ajustados pelos modelos de Ostwald-deWaele (Lei-da-Potência) (Eq. 1) e Herschel-Bulkley (Eq. 2). Valores de viscosidade aparente foram ajustados por uma equação do tipo Arrhenius (Eq. 3).

$$
\begin{gathered}
\tau=\mathrm{K} \gamma^{\mathrm{n}} \\
\tau=\tau_{\mathrm{OH}}+\kappa_{\mathrm{H}} \gamma^{\mathrm{n}_{\mathrm{H}}} \\
\eta=\eta_{\mathrm{o}} \exp \left(\frac{\mathrm{E}_{\mathrm{a}}}{\mathrm{RT}}\right)
\end{gathered}
$$

em que:

$\tau$ - tensão de cisalhamento, $\mathrm{Pa}$

$\mathrm{K}$ - índice de consistência, $\mathrm{Pa} \mathrm{S}^{\mathrm{n}}$

$\gamma$ - taxa de deformação, $\mathrm{s}^{-1}$

$\mathrm{n}$ - índice de comportamento do fluido, adimensional

$\tau_{0 \mathrm{H}}$ - tensão inicial de Herschel-Bulkley, Pa

$\kappa_{\mathrm{H}}$ - índice de consistência de Herschel-Bulkley, Pa s ${ }^{\mathrm{n}}$

$\mathrm{n}_{\mathrm{H}}$ - índice de comportamento de fluido, adimensional

$\eta$ - viscosidade, $\mathrm{Pa} \mathrm{s}$ 
$\mathrm{h}_{0}$ - constante, $\mathrm{Pa} \mathrm{s}$

$\mathrm{E}_{\mathrm{a}}$ - energia de ativação, $\mathrm{J} \mathrm{g}^{-1} \mathrm{~mol}^{-1}$

$\mathrm{R}$ - constante universal gases, $8,14 \mathrm{~J} \mathrm{~kg}^{-1} \mathrm{~K}^{-1}$

$\mathrm{T}$ - temperatura absoluta, $\mathrm{K}$

\section{RESULTADOS E DISCUSSÃO}

\section{Modelo de Ostwald-de-Waele (Lei da Potência)}

Apresentam-se, na Tabela 1, os valores dos parâmetros de ajuste do modelo de Ostwald-de-Waele (Lei da Potência) para o mel da florada silvestre, nas temperaturas de 20 a $40{ }^{\circ} \mathrm{C}$. Constata-se que o modelo apresentou bom ajuste aos dados experimentais e valores do coeficiente de determinação $\left(\mathrm{R}^{2}\right)$ acima de 0,99 e qui-quadrado $\left(\chi^{2}\right)$ próximos a zero. Os valores do índice de consistência $(\mathrm{K})$ e do índice de comportamento do fluido (n) decresceram com o aumento da temperatura. De acordo com Steffe (1996) quando o valor de K é maior que zero e o valor de $\mathrm{n}$ está entre $0<\mathrm{n}<1$, o fluido é considerado não-newtoniano e pseudoplástico.

Tabela 1. Parâmetros de ajuste do modelo de Ostwald-deWaele (Lei da Potência) do mel da florada de silvestre

\begin{tabular}{ccccccc}
\hline $\mathbf{T}\left({ }^{\circ} \mathbf{C}\right)$ & $\left.\mathbf{K ~} \mathbf{~ ( P a ~ s}^{\mathbf{n}}\right)$ & $\mathbf{n}$ & Erro $(\mathbf{K})$ & Erro $(\mathbf{n})$ & $\chi^{2}$ & $\mathbf{R}^{2}$ \\
20 & 5,74791 & 0,97866 & 0,00054 & 0,00100 & 0,00016 & 0,99997 \\
25 & 5,02023 & 0,96647 & 0,00003 & 0,00030 & 0,00280 & 0,99996 \\
30 & 4,61375 & 0,95594 & 0,00001 & 0,00000 & 0,00166 & 0,99977 \\
35 & 1,21197 & 0,93851 & 0,00011 & 0,00010 & 0,00412 & 0,99998 \\
40 & 0,56632 & 0,93515 & 0,00002 & 0,00027 & 0,00240 & 0,99995 \\
\hline
\end{tabular}

\section{Modelo de Herschel-Bulkley}

$\mathrm{Na}$ Tabela 2 se encontram os valores dos parâmetros do modelo de Herschel-Bulkley e os parâmetros estatísticos: erro, coeficiente de determinação $\left(\mathrm{R}^{2}\right)$ e qui-quadrado $\left(\chi^{2}\right)$. Podese observar que este modelo empírico de comportamento reológico apresentou um excelente ajuste aos dados experimentais, com todos os valores do coeficiente de determinação iguais a 0,999 . Verifica-se, dos resultados obtidos, que o índice de consistência $\left(\mathrm{K}_{\mathrm{H}}\right)$, o índice de comportamento do fluido $\left(\mathrm{n}_{\mathrm{H}}\right)$ e a tensão de cisalhamento inicial $\left(\tau_{\mathrm{OH}}\right)$ decresceram com o aumento da temperatura. Confirma-se, então, que o mel analisado apresentou comportamento não-newtoniano, com característica pseudoplástica, conforme se verifica pelos valores de $n_{H}$ variando de 0,92 a 0,99 e que, conforme se baixou

Tabela 2. Parâmetros de ajuste do modelo de HerschelBulkley do mel da florada de silvestre

\begin{tabular}{|c|c|c|c|c|c|c|c|c|}
\hline $\begin{array}{c}\mathrm{T} \\
\left({ }^{\circ} \mathrm{C}\right)\end{array}$ & $\tau_{0 \mathrm{H}}(\mathrm{Pa})$ & $\begin{array}{c}\kappa_{H} \\
\left(\mathrm{~Pa} \cdot \mathrm{s}^{\mathrm{n}}\right)\end{array}$ & $n_{H}$ & $\begin{array}{l}\text { Erro } \\
\left(\tau_{\text {OH }}\right)\end{array}$ & $\begin{array}{l}\text { Erro } \\
\left(\kappa_{\mathrm{H}}\right)\end{array}$ & $\begin{array}{l}\text { Erro } \\
\left(n_{H}\right)\end{array}$ & $\chi^{2}$ & $\mathbf{R}^{2}$ \\
\hline 20 & 0 , & 5,32115 & 0,9908 & 0,00011 & 0,00036 & 0,000 & 0,0 & $0, \subseteq$ \\
\hline 25 & 0 , & 4,41 & 0 , & 0, & 0 , & 0,001 & 0 , & 0 , \\
\hline 30 & 0,13299 & 1,17456 & 0,9559 & 0,0 & 0,00027 & 0,003 & 0,00000 & 0,99998 \\
\hline 35 & 0,00815 & 0,74834 & 0,9359 & 0,00013 & 0,00063 & 0,000 & 0,00000 & 0,99999 \\
\hline 40 & 0,00092 & 0,56419 & 0,9216 & 0,00252 & 0,00001 & 0,001 & 0,00001 & 0,99998 \\
\hline
\end{tabular}

a temperatura, $\mathrm{n}_{\mathrm{H}}$ aproximou-se de 1 . Tal resultado se enquadra na variabilidade do comportamento reológico dos méis reportado em literatura, como em Abu-Jdayil et al. (2002), que caracterizaram o mel como fluido newtoniano, e Muller (1977) que, estudando mel procedente de flores de eucalipto (Eucalyptus ficifolia), afirmou que o mel tem comportamento nãonewtoniano e reopético. Diversos trabalhos realizados com méis discutem a sua natureza reológica, embora a maioria afirme tratar-se de um fluido newtoniano; alguns outros obtiveram valores de $\mathrm{n}$ (índice de comportamento do fluido) inferiores ou mesmo superiores à unidade (Bath \& Singh, 1999).

Apesar dos bons ajustes proporcionados pelo modelo de Ostwald-de-Waele, este foi superado pelo modelo de Herschel-Bulkley, que ajustou os pontos experimentais com coeficientes de determinação ainda maiores.

Apresentam-se, na Figura 1, os pontos experimentais de tensão de cisalhamento em função da taxa de deformação do mel da florada de silvestre, com ajustes pela de equação de Herschel-Bulkley, nas temperaturas de 20 a $40{ }^{\circ} \mathrm{C}$. Observa-se que as posições das curvas demonstram viscosidades distintas entre as amostras, identificando-se que a temperatura influenciou o comportamento viscoso do mel, com o aquecimento provocando diminuições nas viscosidades. Pereira et al. (2003) também verificaram o mesmo comportamento para o mel de uruçu (Melipona scutellaris L.); este efeito não se mostrou progressivo uma vez que as curvas para 20 a $30{ }^{\circ} \mathrm{C}$ compuseram um grupo distanciado em relação às curvas a 35 e $40^{\circ} \mathrm{C}$.

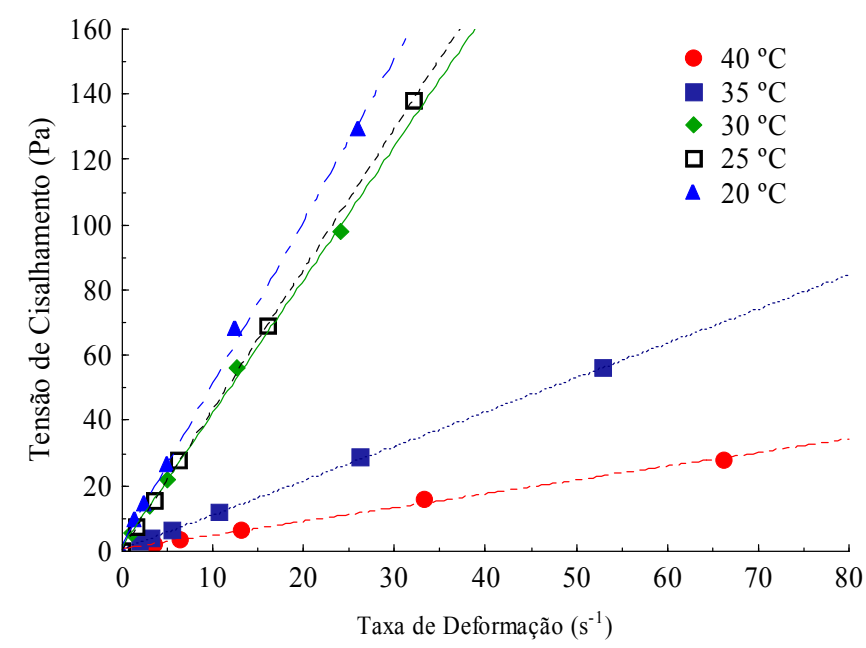

Figura 1. Relação entre a tensão de cisalhamento e a taxa de deformação com ajuste pelo modelo de Herschel-Bulkley do mel da florada de silvestre nas temperaturas de 20 a $40^{\circ} \mathrm{C}$

\section{Viscosidade aparente}

Na Figura 2 estão apresentadas as viscosidades aparentes do mel da florada de silvestre, para velocidades de rotação variando entre 0,5 e $100 \mathrm{rpm}$. Observam-se reduções nas viscosidades aparentes provocadas pelo aumento de temperatura e pela velocidade de rotação. Com o aumento da temperatura, a viscosidade do mel foi reduzida em cerca de $80 \%$ entre 20 e 40 ${ }^{\circ} \mathrm{C}$; já as reduções da viscosidade com o aumento da velocidade de rotação foram menores, oscilando de 13 a 33\%. 
Na Figura 2 é possível observar as reduções de viscosidade aparente com o aumento da velocidade de rotação em todas as temperaturas, evidenciando o caráter pseudoplástico das amostras. As curvas referentes às temperaturas de 20 e $25^{\circ} \mathrm{C}$ se destacam das demais, em razão de seus valores de viscosidade aparente superarem entre quatro a cinco vezes, aproximadamente, as viscosidades aparentes nas demais temperaturas; já Sopade et al. (2002) verificaram viscosidades em méis australianos entre seis e nove vezes maiores, na temperatura de $21{ }^{\circ} \mathrm{C}$ em relação à de $40{ }^{\circ} \mathrm{C}$.

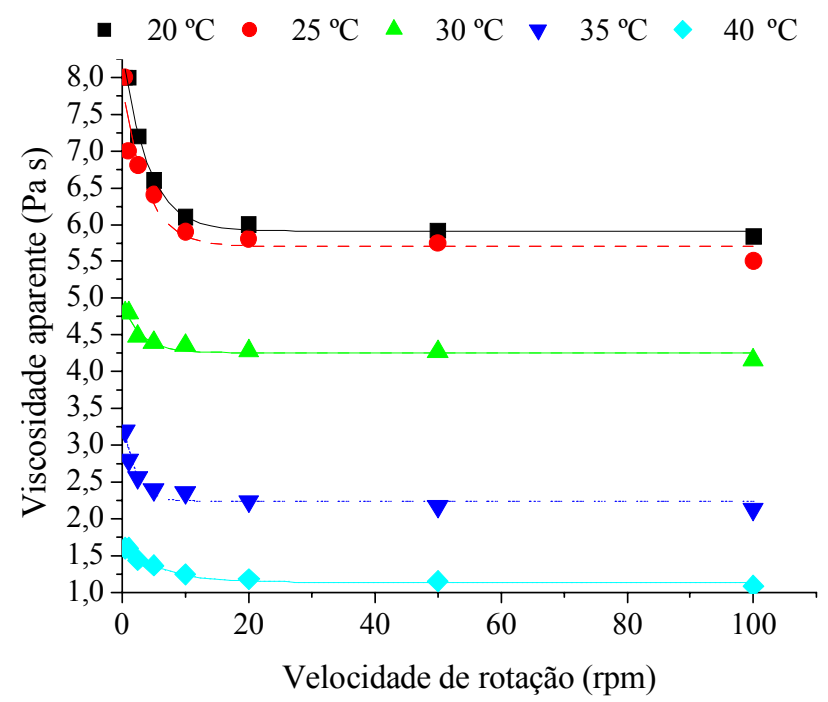

Figura 2. Relação entre a viscosidade aparente e a velocidade de rotação do mel da florada de silvestre, nas temperaturas de 20 a $40^{\circ} \mathrm{C}$

\section{Equação de Arrhenius}

Os valores de viscosidade aparente foram ajustados por uma equação do tipo Arrhenius, estando as energias de ativação e as constantes $\left(\eta_{0}\right)$ resultantes do ajuste, apresentados na Tabela 3. Pelo valor dos coeficientes de determinação $\left(\mathrm{R}^{2}\right)$ acima de 0,89 pode-se observar que a equação apresentou um bom ajuste aos dados de viscosidade aparente. Verifica-se, também, que a energia de ativação variou de 62,16 a $65,25 \mathrm{~kJ} \mathrm{~g}^{-1} \mathrm{~mol}^{-1}$. Alvarado (2001) determinou uma energia de ativação para o mel de $84,39 \mathrm{~kJ} \mathrm{~mol}^{-1}$, para temperaturas entre

Tabela 3. Valores da energia de ativação $\left(E_{a}\right)$ e viscosidade do mel da florada silvestre Arrhenius (viscosidade $\eta_{0} x$ temperatura)

\begin{tabular}{cccc}
\hline $\begin{array}{c}\text { Velocidade de rotação } \\
(\mathbf{r p m})\end{array}$ & $\eta_{0}(\mathbf{P a ~ s})$ & $\mathbf{E}_{\mathbf{a}}\left(\mathbf{k J ~ ~ ^ { - 1 }} \mathbf{~ m o l}^{-1}\right)$ & $\mathbf{R}^{2}$ \\
0,5 & $6,6131 \times 10^{-11}$ & 62,72 & 0,9161 \\
1,0 & $6,1039 \times 10^{-11}$ & 62,79 & 0,9480 \\
2,5 & $3,9705 \times 10^{-11}$ & 63,68 & 0,9315 \\
5,0 & $5,3230 \times 10^{-11}$ & 62,79 & 0,9161 \\
10,0 & $6,4549 \times 10^{-11}$ & 62,16 & 0,8944 \\
20,0 & $3,3999 \times 10^{-11}$ & 63,70 & 0,8945 \\
\hline 50,0 & $2,6390 \times 10^{-11}$ & 64,30 & 0,8914 \\
100,0 & $1,7507 \times 10^{-11}$ & 65,25 & 0,8931 \\
\hline
\end{tabular}

40 e $70^{\circ} \mathrm{C}$, relatando que este valor é alto, indicando que a viscosidade do mel de abelhas é altamente dependente da temperatura. Mossel et al. (2000) fazendo uma comparação entre méis australianos, determinaram energias de ativação na velocidade de rotação de $50 \mathrm{rpm}$ variando de 66,31 a 99,29 kJ $\mathrm{g}^{-1} \mathrm{~mol}^{-1}$ para uma faixa de temperatura entre 1 e $40{ }^{\circ} \mathrm{C}$.

\section{CONCLUSÕES}

1. O mel elaborado com florada predominante de silvestre apresentou comportamento pseudoplástico.

2. As equações da Lei da Potência e de Herschel-Bulkley resultaram em bons ajustes aos dados experimentais de tensão de cisalhamento, em função da taxa de deformação.

3. O aumento de temperatura de 20 a $40{ }^{\circ} \mathrm{C}$ reduziu a viscosidade aparente em mais de $90 \%$.

4. Os valores de viscosidade aparente foram satisfatoriamente ajustados por uma equação do tipo Arrhenius.

\section{LITERATURA CITADA}

Abu-Jdayil, B.; Ghzawi, A.A.; Al-Malah, K.I.M. Heat effect on rheology of light and dark-colored honey. Journal of Food Engineering, New York, v.51, n.1, p.33-38, 2002.

Alcoforado Filho, F.G. Sustentabilidade do semi-árido através da apicultura. In: Congresso Brasileiro de Apicultura, 13, 1998, Salvador. Resumos... Salvador: CBA, 1998. p.61.

Alvarado, J.D. Viscosidade. In: Alvarado, J.D.; Aguilera, J.M. Métodos para medir propiedades físicas em industrias de alimentos. Zaragoza: Acribia, 2001. cap.1, p.61-87.

Balbach, A.; Boarim, D. As frutas na medicina natural. 1.ed. São Paulo: Vida Plena, 1992. 308p.

Bath, P.K.; Singh, N. A comparison between Helianthus annuus and Eucalyptus lanceolatus honey. Food Chemistry, London, v.67, n.4, p.389-397, 1999.

Brasil. Instrução normativa $n^{\circ} 11$, de 20 de outubro de 2000. Estabelece o regulamento técnico de identidade e qualidade do mel. Diário Oficial [da] República Federativa do Brasil, Poder Executivo, Brasília, 23 out. 2000. Seção 1, p.16-17.

Campos, M.G.R. Contribuição para o estudo do mel, pólen, geléia real e própolis. Boletim da Faculdade de Farmácia de Coimbra, Coimbra, v.11, n.2, p.17-47, 1987.

Crane, E. O livro do mel. São Paulo: Nobel, 1983. 226p.

Hooper, T. Guia do apicultor. 2.ed. Sintra: Europa-América, 1981. 269p.

Ibarz, A.; Gonçalves, C.; Explugas, S. Rheology of clarified passion fruit juices. Fruit Processing, New Delhi, v.6, n.8, p.330-333, 1996.

IBGE - Instituto Brasileiro de Geografia e Estatística. Produção da pecuária municipal. Brasil: IBGE, 2003. 29p.

R. Bras. Eng. Agríc. Ambiental, v.11, n.2, p.190-194, 2007. 
Marques, A.N.; Wiese, H. Os produtos das abelhas. In: Wiese, H. Nova apicultura. Porto Alegre: Agropecuária, 1983. cap.14, p.409-421.

Mitschka, P. Simple conversion of Brookfield RVT: readings into viscosity functions. Rheologica: Acta, Prague, v.21, n.2, p.207-209, 1982.

Mossel, B.; Bhandari, B.; D’Arcy, B.; Caffin, N. Use of an Arrhenius model to predict rheological behaviour in some Australian honeys. Lebensmittel-Wissenschaft and Technologie, New York, v.33, n.8, p.545-552, 2000.

Muller, H.G. Introducción a la reologia de los alimentos. Zaragoza: Acribia, 1977. 174p.
Pereira, E.A.; Queiroz, A.J.M.; Figueirêdo, R.M.F. Comportamento reológico de mel da abelha urucu (Melipona scutellaris L.). Revista Ciências Exatas e Naturais, Guarapuava, v.5, n.2, p.179-186, 2003.

Sopade, P.A.; Halley, P.; Bhandari, B.; D’Arcy, B.; Doebler, C.; Caffin, N. Application of the Williams-Landel-Ferry model to the viscosity-temperature relationship of Australian honeys. Journal of Food Engineering, New York, v.56, n.1, p.67-75, 2002.

Steffe, J.F. Rheological methods in food process engineering. 2.ed. Michigan: Freeman Press, 1996. 418p. 